\title{
Discoid lupus erythematosus in Iraqi patients: a clinical and histopathological study
}

Mohammed Kadhim Al-Hattab, MBChB; Makram Al-Waiz, MBChB, DDSc, Phd

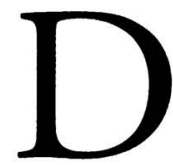
iscoid lupus erythematosus (DLE) is a relatively benign disorder of the skin, most frequently involving the face, and characterized by various-sized, reddish, well-defined scaly patches, which tend to heal with atrophy, scarring and pigmentary changes. ${ }^{1}$ It has been proposed that genetic factors and somatic mutations are implicated in the pathogenesis of the disease. ${ }^{2}$ Classical DLE lesions, most frequently of the circumscribed or discoid type, occur most commonly on sun-exposed areas of the body, ${ }^{3}$ particularly on the cheeks, the bridge of the nose, the ears, and the side of the neck and scalp. ${ }^{1}$ Early classical DLE lesions typically evolve into sharply demarcated, coin-shaped erythematous plaques covered by a prominent, adherent scale that extends into the orifices of dilated hair follicles. ${ }^{4}$ Early DLE lesions typically expand with erythema and hyperpigmentation at the periphery, leaving atrophic central scarring, telangiectasia and hypopigmentation. ${ }^{4}$ Localized DLE lesions occur only on the head or neck, whereas generalized DLE lesions occur both above and below the neck. ${ }^{4}$ Other variants of DLE include verrucous (hypertrophic) LE, LE profundus, LE panniculitis, mucosal DLE, LE-LP (lupus erythematosus-lichen planus) overlap syndrome, LE telangiectoides and chilblain LE. ${ }^{1,4,5}$ Laboratory abnormalities are found in about $55 \%$ of patients. ${ }^{5}$ The presence of persistently abnormal laboratory tests findings and a high titre of ANA in DLE appears to be an indication of the potential for SLE to develop. ${ }^{6}$ The aim of this study was to shed light on the various clinical and histopathological aspects of DLE among Iraqi patients.

\section{Patients and methods}

We studied 54 patients with DLE. A full history from each patient was recorded, including age, sex, age of onset and duration of the disease, and precipitating and aggravating factors. A thorough physical examination was conducted to record the site of the lesion, the symmetry and distribution of the lesions, and to determine whether bilateral or unilateral and different clinical variants of the disease were present. Laboratory investigations included ANAs (anti-nuclear antibodies) (with titer), anti-ds-DNA antibodies, a complete blood picture and ESR (erythrocyte sedimentation rate), and a VDRL (Venereal Disease Research Laboratory) test (with titer) for false positive serological tests associated with DLE. A general urine examination was also performed for each patient. Biopsies were taken from 32 patients for

\author{
From the Department of Dermatology and Venereology, \\ Baghdad Teaching Hospital, College of Medicine, \\ University of Baghdad \\ Baghdad, Iraq \\ Correspondence to: \\ Professor Makram Al-Waiz \\ P.O. Box 61269 Medical Collection Post Office \\ Postal Code Number 12114 \\ Bab Al-Mua'dham \\ Baghdad \\ Iraq \\ Email:makram55@uruklink.net
}

Accepted for publication: March 2003

Ann Saudi Med 2004;24(4):289-292

histopathological examination by hematoxylin and eosin staining.

\section{Results}

The 54 patients ranged in age from 13 to 70 years (mean \pm SD, $36 \pm 11.8$ years), while age of onset averaged between 7 to 68 years (mean $\pm S D, 30.2 \pm 11.4$ years). The duration of disease ranged from 1 month to 30 years (median, 3 years). There were 34 females $(63 \%)$ and 20 males $(37 \%)$ for a sex ratio of 1.7:1.

The clinical variants of the disease in our patients are shown in Table 1. A variant of disseminated leukoderma or vitiligo-like DLE, which involved the face, most of the trunk and extremities with scarring alopecia of the scalp was seen in one patient (1.8\%) (Figure 1). Hypertrophic DLE with a warty-like plaque at the extensor surface of the right forearm was seen in one of the disseminated classical DLE patients. Table 2 shows the sites of involvement. The cheeks and the nose were the commonest sites of involvement ( $76 \%$ and $57.4 \%$, respectively). The scalp was affected in $48 \%$ of patients. Scarring alopecia was seen in $42.5 \%$ and the vertex was the most commonly affected site $(37 \%)$. Non-scarring hair loss was seen in $5.5 \%$. The oral cavity was involved in $11 \%$ of cases. The lesions were whitish, atrophic patches located on the hard palate and buccal mucosa. Two patients with oral ulcers progressed to SLE. The nasolabial folds were not affected in all patients with DLE. Nails and nail folds were also not affected. 
Table 1. Clinical presentation in 54 patients with discoid lupus erythematosus (DLE).

\begin{tabular}{lc} 
Clinical variant of DLE & No. cases (\%) \\
Localized classical & $24(44.4)$ \\
Localized atrophic & $12(22.2)$ \\
Disseminated classical & $8(14.8)$ \\
Disseminated atrophic & $8(14.8)$ \\
Lupus profundus (panniculitis) & $6(11)$ \\
Hypertrophic & $1(1.8)$ \\
Disseminated leukoderma-like & $1(1.8)$ \\
\hline
\end{tabular}

Table 2. Sites of lesions in 54 patients with DLE

\begin{tabular}{lc}
\hline Site & No. cases (\%) \\
\hline Cheeks & $41(76)$ \\
Nose & $31(57.4)$ \\
Ears & $25(46)$ \\
Around ears & $21(39)$ \\
Forehead & $6(11)$ \\
Mandibles & $7(13)$ \\
Lips & $11(20.4)$ \\
Periorbital & $7(13)$ \\
Chin & $6(11)$ \\
Oral mucosa & $6(11)$ \\
Scalp & $26(48)$ \\
Neck & $2(3.7)$ \\
Trunk & $14(26)$ \\
Arms & $11(20.4)$ \\
Legs & $1(1.8)$ \\
\hline *Nearly all patients had more than one site of involvement \\
of DLE lesions.
\end{tabular}

Anti-nuclear antibodies (ANA) were positive in 13 patients (24\%) with a titre ranging between $>1: 40$ to $>1: 80$. The patterns of ANA reactions were speckled in 9 patients $(16.6 \%)$, homogenous in 3 patients $(5.6 \%)$ and nucleolar in 1 patient (1.8\%). Anti-ds-DNA antibodies were positive in 6 patients (11\%). Two of the 3 patients who started as DLE and changed to SLE had positive anti-ds-DNA antibodies. The VDRL test was false positive in 5 patients with a titer ranging between 1 : 8 to $1: 16(9.2 \%)$. Hemoglobin levels ranged between 9 to 10 $\mathrm{g} / \mathrm{dL}$ in 9 patients $(16.6 \%)$. Leukopenia $(<3000 / \mathrm{mm} 3)$ was found in 5 patients (9.2\%); 2 of 3 patients who changed to SLE had leukopenia in serial measurements. ESR levels were high, ranging from 45 to $115 \mathrm{~mm} /$ hour in 10 patients (18.5\%). GUE showed positive granular and epithelial casts in 2 patients (3.7\% ) (Table 3). The histopathological findings in 32 patients with different clinical variants of the disease are shown in Table
Table 3. Laboratory abnormalities in 54 patients with DLE

\begin{tabular}{lc}
\hline Parameter & No. cases (\%) \\
\hline Positive ANA & $13(24)$ \\
Positive anti-ds-DNA antibody & $6(11)$ \\
False positive VDRL test & $5(9.2)$ \\
Anemia & $9(16.6)$ \\
Leukopenia & $5(9.2)$ \\
High ESR levels & $10(18.5)$ \\
$\begin{array}{l}\text { Granular or epithelial cases } \\
\text { in GUE }\end{array}$ & $2(3.7)$ \\
\hline
\end{tabular}

Table 4. Histopathological findings in 32 patients with DLE lesions.

\begin{tabular}{lc|}
\hline Feature & No. cases (\%) \\
\hline Hyperkeratosis & $26(81.2)$ \\
Follicular plugging & $28(87.5)$ \\
Parakeratosis & $4(12.5)$ \\
Atrophy of epidermis & $21(65.6)$ \\
Irregular acanthosis & $4(12.5)$ \\
\hline Normal thickness epidermis & $7(21.8)$ \\
Papillomatosis & $2(6.2)$ \\
Focal basal liquefaction & $23(71.8)$ \\
Diffuse basal liquefaction & $2(6.2)$ \\
\hline Pigmentary incontinence & $11(34.3)$ \\
Telangiectasia & $11(34.3)$ \\
Colloid bodies & $5(15.6)$ \\
Patchy, periappendigeal and & $24(75)$ \\
perivascular inflammatory infiltrate & $3(9.4)$ \\
Sparse upper dermal infiltrate & $1(3)$ \\
Granuloma-like inflammatory & $2(6.2)$ \\
infiltrate & Panniculitis
\end{tabular}

4. Figure 2 shows the histopathological features of classical DLE. A 50 year-old female patient with a scar due to previous localized atrophic DLE in a butterfly distribution on the face of 20 years duration developed squamous cell carcinoma in the previous year on the part of the scar located on the upper lip. No metastasis to regional lymph nodes was noticed (Figure 3 ).

\section{Discussion}

DLE is primarily a disease of adult life with the average time of onset in the fourth decade, with a female:male ratio of about 2 : 1.3 We found that the disease occurred commonly in adults, mainly in the fourth decade of life with a female:male ratio about 1.7:1. This finding is comparable to those reported in the literature. ${ }^{3}$

The clinical variants and the morphology of skin lesions of the patients in the present study were comparable to what 


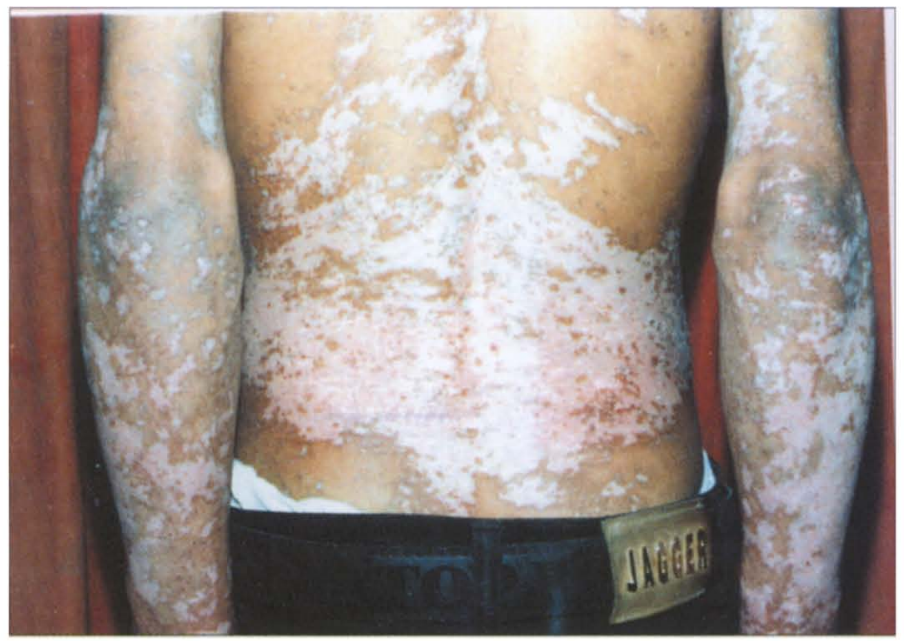

Figure 1. Disseminated leukopenia or vitiligo-like discoid lupus erythematosus (DLE). Multiple and extensive hypopigmented atrophic patches affecting the back and extensors of the forearm.

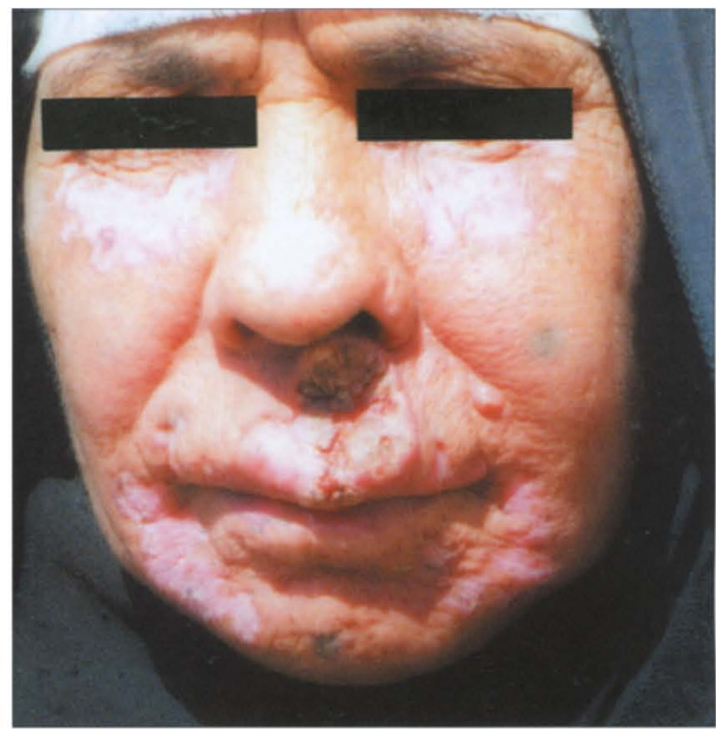

Figure 3. Squamous cell carcinoma complicating the scar of localized atrophic DLE involving the upper lip.

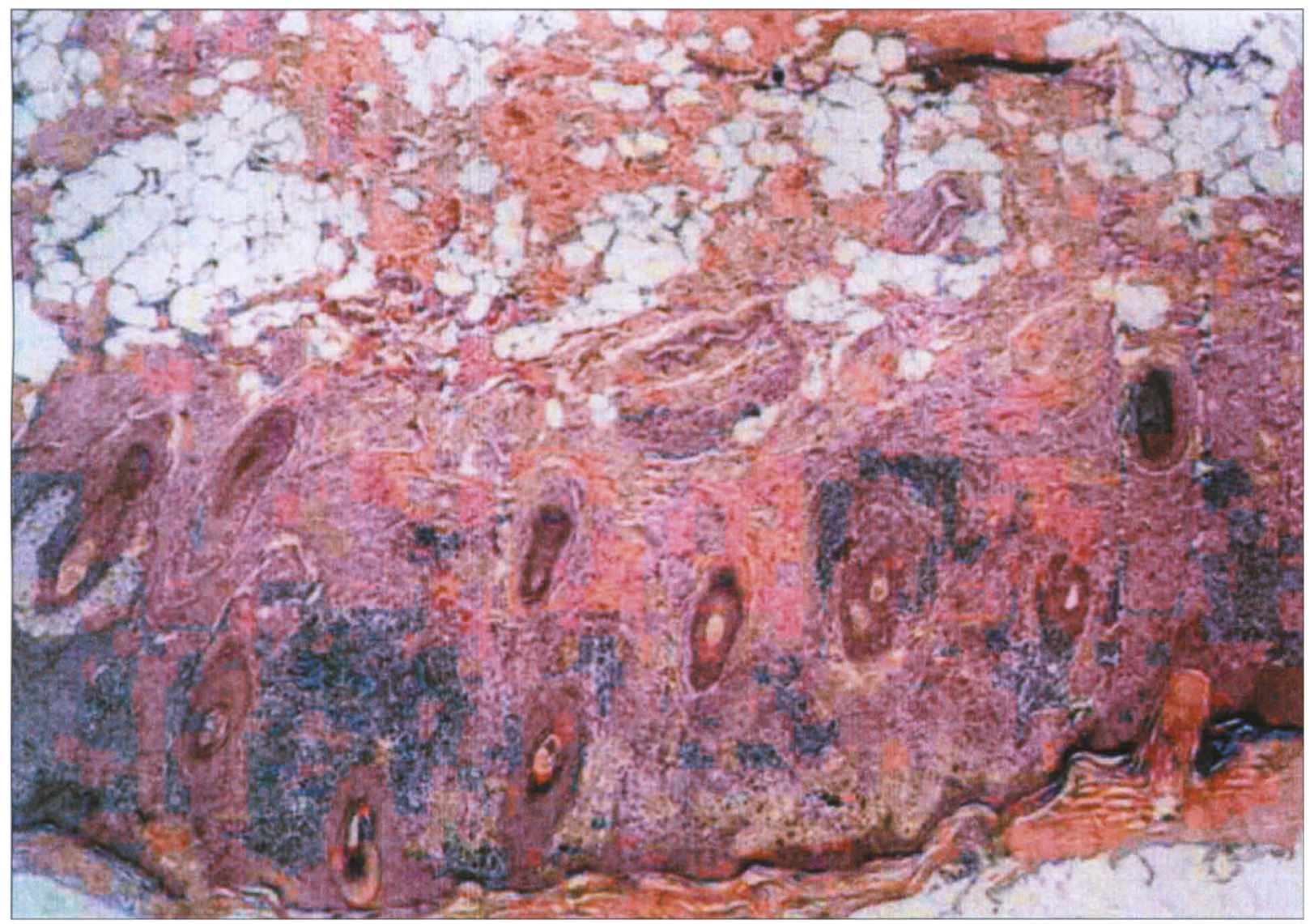

Figure 2. Histopathology of localized classical DLE showing the typical histopathological features of DLE: hyperkeratosis, follicular plugging, atrophic epidermis, and patchy and periappendegeal lymphoid inflammatory cells, mainly in the upper dermis (H\&E, $x 40)$. 
of the patients in the present study were comparable to what has been published. ${ }^{1,4}$ However, a variant of disseminated DLE was found. This variant, a leukoderma or vitilgo-like form, misdiagnosed earlier as a case of vitiligo, was seen in one patient $(1.8 \%)$. We found that the scalp was involved in $48 \%$ of patients with scarring hair loss in $42.5 \%$ and nonscarring hair loss in $5.5 \%$. In the literature, scalp involvement has been similarly affected. ${ }^{3,4}$ In this study, as well as in a previous Iraqi study concerning scarring alopecia in Iraqi patients, ${ }^{7}$ active DLE lesions on the face associated with scarring alopecia of the scalp suggest that the scalp could be involved early in the disease process. This finding has not been reported before. The present work showed that oral mucosa was involved in $11 \%$ with oral ulceration in $3.7 \%$ of patients. This was comparable to what has been described in the literature. ${ }^{3}$ Ears are commonly affected in DLE. Either one ear or bilateral involvement was seen in $46 \%$ of patients. Lesions were located on the rim or the external auditory meatus of the ear. Ear involvement was seen even in those patients who covered their ears continuously when outdoors (e.g., wearing a veil in females), an observation that may reinforce the autoimmune pathogenesis of the disease. Therefore, patients with DLE on the face should have their ears examined.

Previous studies have reported that ANAs were found in $30 \%$ to $35 \%$ of patients with DLE..$^{1,3}$ The homogenous type of antinuclear factor was twice as frequent as the 'speckled' type. ${ }^{1.3}$ We showed that ANAs were positive in $24 \%$, with the 'speckled' pattern of antinuclear factor being three times as frequent as the 'homogenous' type. One patient had a nucleolar pattern. This study demonstrated that ANAs were more common in the disseminated type, in disease with oral involvement, in patients with scarring alopecia, and in patients who had the disease for an extended period of time. Anti-ds-DNA antibodies were positive in $11 \%$ of patients. This was comparable to what has been reported in the literature. ${ }^{8,9}$ These antibodies were found to be positive especially in those patients who started with DLE and transformed to SLE. The risk of a patient with DLE developing overt SLE is small. Transformation varies from $1.3 \%$ to $6.5 \% .{ }^{5,6,10}$ The risk is higher in patients with disseminated DLE than in DLE confined to the head and neck. ${ }^{5,6,10}$ We found that the percentage of patients transforming to SLE was 5.5\%, which is comparable to rates reported in the literature. ${ }^{5,6,10}$

The incidence of neoplastic changes in DLE was about $1.8 \%$, which is comparable to the incidence of $3.3 \%$ noticed in a series of 120 white patients with DLE. ${ }^{11}$ The squamous cell carcinoma in a scar on the upper lip one patient had been present for about 3 months with no metastasis to regional lymph nodes. The patient had a duration of DLE of about 20 years. The histopathological features of different clinical variants of DLE were similar to what has been reported. ${ }^{1,12}$ Although it is well known that parakeratosis is not conspicuous and may be absent, ${ }^{12}$ we showed that parakeratosis was present in $12.5 \%$ of specimens. This discrepancy could be attributed to superimposition of other inflammatory disorders such as dermatitis or malignancy. DLE seems not uncommon in Iraq. No previous study has evaluated the disease in our country so our report is a first step towards shedding light on this important subject.

\section{References}

1. Rowell NR,Goodfield MJD. The connective tissue diseases. In: Champion RH, Burton JL, Burns DA, Breathnach SM, eds. Rook/Wilkinson/Ebling Textbook of Dermatology. 6th ed. Oxford: Blackwell scientific publications; 1998: 2437-2459.

2. Burch PRJ, Rowell NR. Lupus erythematosus: Analysis of the sex and age distributions of the discoid and systemic forms of the disease in different countries. Acta Dermatovener (Stockholm). 1970; 50: 293-301.

3. Kathllen Clark S. Cutaneous lupus erythematosus, Recognition of its many forms. Postgrad Med. 1986; 79: 195-200.

4. Sontheimer RD. Lupus Erythematosus. In: Fitzpatrick's $T B$, Irwin $M$, Freedberg, Eisen $A Z$, Austen KW, Gold Smith LA, Katz SI, Thomas BF, eds.
Dermatology in General Medicine. 5th ed. New York: McGraw-Hill Book Co. 1999;1993 - 2009.

5. Callen JP, Provost TT, Tuffanelli DL, Kohler PF, Condem Jj. Connective tissue disease. In: Richard BO, William DJ, Timothy GB, eds. Andrews' Diseases of the Skin, Clinical Dermatology. 9th ed. W.B. Saunders Co. 2000; 172-185.

6. Rowell NR. Laboratory abnormalities in the diagnosis and management of lupus erythematosus. $\mathbf{B r} \mathbf{J}$ Dermatol. 1971; 84: 210-216.

7. Al-Shahwani FA, Al-Waiz MM. Scarring alopecia, clinical study and its frequency in outpatients clinic in Iraqi patients. Iraqi J Com Med. 2001; 14: 110-3.

8. Davis P, Atkins B, Hughes GRV. Antibodies to native DNA in DLE. Br J Dermatol. 1974; 91:175-181.
9. Prystowsky SD, Gilliam JN. Discoid lupus erythematosus as part of a larger disease spectrum. Arch Dermatol. 1975; 111:1448-1452.

10. Millard LG, Rowell NR. Abnormal laboratory test results and their relationship to prognosis in discoid lupus erythematosus. Arch Dermatol. 1979;115: 1055-1058. 11. Millard LG, Barker DJ. Development of squamous cell carcinoma in chronic discoid lupus erythematosus. Clin Exp Dermatol. 1978; 3: 161.

12. Jaworsky $C$. Connective tissue diseases. In: Elder $D$, Elenitsas R, Jaworsky C, Johnson Jr, B eds. Lever's Histopathology of the skin. 8th ed. Philadelphia: LippincottRaven publishers. 1997;253-268. 